\title{
Oral Hygiene and Maintenance of Periodontal Support*
}

\author{
S. P. Ramfjord, † E. C. Morrison, F. G. Burgett, R. R. Nissle, \\ R. A. Shick, G. J. Zann, and J. W. Knowles \\ Accepted for publication 9 February 1981
}

\begin{abstract}
THE ROLE OF personal plaque control in periodontal maintenance care was studied in 78 patients who had undergone periodontal therapy and were on 3-month recall for prophylaxis over 8 years. Variations in pocket depth and attachment levels were related to individuals with plaque scores above and below the median. The results also were analyzed by comparing the $25 \%$ of the sample having the lowest plaque scores with the $25 \%$ having the highest scores over 7 years of maintenance care. Students $t$ test was used. It was found that personal oral hygiene as expressed in plaque scores was not critical for maintenance of post-treatment pocket depth and attachment levels in patients with professional tooth cleaning every 3 months. The initial post-treatment reductions in pocket depth and variations in attachment levels were more favorable in patients with good than with poor oral hygiene, but, these differences were not significant after 3 to 4 years of maintenance care.
\end{abstract}

It has been established that lack of adequate supragingival plaque removal over time will result in gingivitis. ${ }^{1}$ There also is ample evidence that lack of plaque removal over long periods may lead to periodontitis both in animals $^{2}$ and in man. ${ }^{3,4}$

Furthermore, periodic removal of plaque and other surface irritants may cure gingivitis ${ }^{l}$ and arrest the progress of periodontitis. ${ }^{5,6}$

However, beyond these established facts there are wide gray zones regarding the impact on periodontal health status of the type, amount, location and duration of plaque, not to mention individual tolerance levels to plaque irritants.

The dictum of a plaque free mouth as a basic requirement for periodontal health and as an ultimate goal for periodontal therapy has been presented convincingly and seemingly supported by evidence from well controlled investigations. ${ }^{7-9}$ Equally well documented has been the inevitable failure of periodontal therapy which is not followed by adequate plaque control. ${ }^{10}$ However, clinical observation and well controlled longitudinal studies ${ }^{11,12}$ also indicate that over a period of several years sizable population groups may maintain clinically measurable attachment levels for their teeth with less than perfect plaque control.

In many children and some adults one may find definite plaque on the teeth without clinical evidence of gingivitis. Furthermore, complete plaque control based

\footnotetext{
* This study was partially supported by United States Public Health Service Grant DE 02731.

$\dagger$ Department of Periodontics, The University of Michigan, School of Dentistry, Ann Arbor, MI 48109.
}

on patient cooperation appears to be unattainable even in selected populations using chemical as well as mechanical means of plaque removal and prevention. ${ }^{13}$

Axelsson and Lindhe's ${ }^{14}$ concept of periodic professional tooth cleaning, and more recent studies on the renewal time required for organisms in periodontal pockets ${ }^{15}$ following episodes of treatment, ${ }^{16,17}$ may explain why the personal oral hygiene status may not have as dominant an effect on periodontal health as previously assumed.

We have reported previously that clinically measurable periodontal attachment levels and pocket depths may be maintained for several years after periodontal treatment in spite of less than perfect plaque control. ${ }^{12}$ However, not until the present paper have we attempted to assess the role that variations in post-treatment plaque scores may play in the response to treatment over time, when the patients are kept on a program of professional toothcleaning (prophylaxis) every 3 months.

\section{MATERIALS AND METHODS}

All patients in the present study were included in our 8-year report in $1979 .{ }^{12}$ However, a few more patients who had completed 6- to 8-year follow-ups since the previous report were included in the present paper. The total number of patients was 78. They all had initial hygienic phase therapy including occlusal adjustment, followed by surgical therapy, and recall with prophylaxis every 3 months. Scorings were repeated annually. The results following all methods of treatment are pooled in the present paper. As in previous reports, pocket depth and clinical attachment levels were grouped in three 
classes (1-3 mm, 4-6 $\mathrm{mm}$ and 7-12 $\mathrm{mm}$ ). Means of measurement within each of these groups were calculated for each patient and the patient's means were used as the basis for the statistical analysis.

Plaque was scored according to the PDI index ${ }^{18}$ but the buccal and lingual aspects were scored separately and the two scores added to give the score for the tooth. Then the individual scores for the teeth were added and divided by the number of teeth to derive a patient mean which was used for the statistical calculations.

Variations in pocket depth and attachment levels for individuals over time were related to plaque scores which were higher or lower than median scores, the assumption being that higher scores represent poorer oral hygiene and that lower scores indicate better oral hygiene.

The initial pocket depth and attachment level were used as baseline for subsequent variations in these parameters over time. The plaque scores at 1 year of followup were used to separate individuals with low or high plaque scores. According to previous reports, plaque scores will not change significantly for an individual with our type of maintenance care. ${ }^{19}$

The students $t$ test was used to test the hypothesis of equal effect of plaque scores above and below the median for the three severity groups of the initial disease based on pocket depth. Since the variations in post-treatment plaque scores were relatively low because of treatment effect and recall for prophylaxis every 3 months, another grouping of the patients was undertaken, with the $25 \%$ of patients with the highest plaque scores in one group and the 25\% with the lowest scores in another group. These scores were then related to variations in pocket depth and attachment levels.

\section{RESULTS}

The reduction in pocket depth for pockets in the 1 to $3 \mathrm{~mm}$ class (Fig. 1), initially and over 8 years, was not significantly different for individuals with plaque scores above or below the median. However, there was a slightly greater initial reduction in pocket depth in the group with lower than median plaque scores (good oral hygiene), and this difference was essentially maintained over 8 years. After year 1 the curves behaved almost identically, indicating no more return of pocket depth
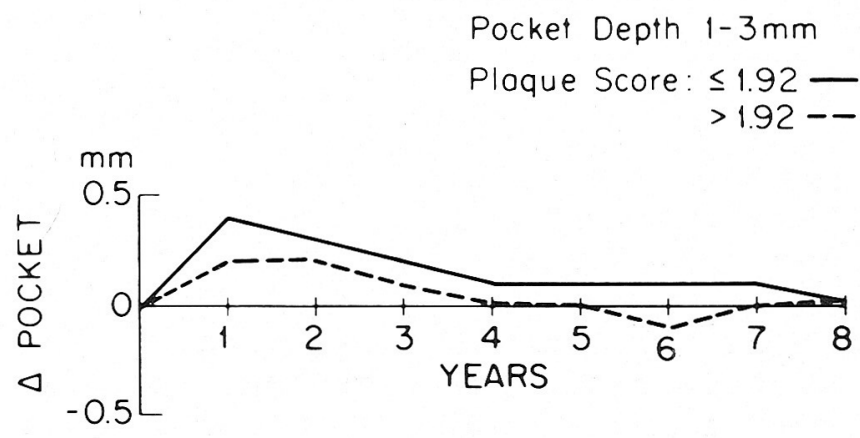

Figure 1. Pocket reduction related to oral hygiene over 8 years.

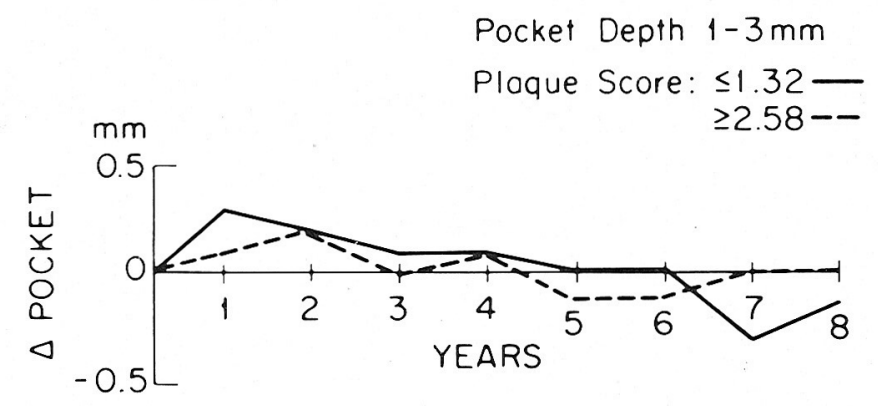

Figure 2. Pocket reduction related to oral hygiene over 8 years.

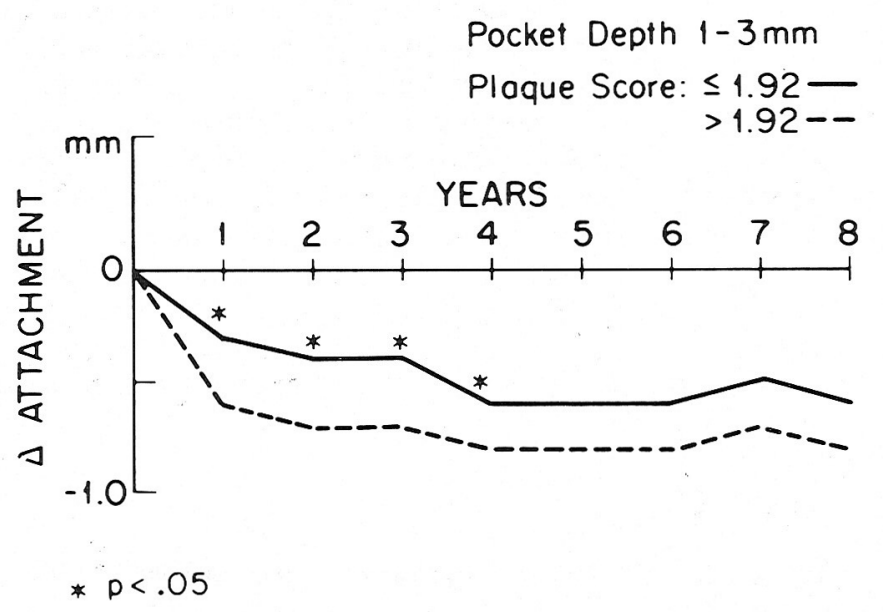

Figure 3. Attachment change related to oral hygiene over 8 years.

with poorer than average oral hygiene than with better than average.

A comparison of the $25 \%$ of patients having the lowest plaque scores with the $25 \%$ having the highest score (Fig. 2) showed no significant differences in pocket depth responses over the 8 years. When variations in attachment levels over time were related to higher or lower than median plaque scores (oral hygiene) (Fig. 3), the initial loss of attachment that we previously described for this group was significantly greater for those with poorer oral hygiene, and this significant difference lasts for 4 years. However, the behavior of the two curves after year 1 was very similar and there was no indication that the poorer oral hygiene leads to a greater loss of attachment after year 1 than the better oral hygiene.

When the individuals with the upper $25 \%$ of plaque scores were compared with the lower $25 \%$ (Fig. 4), the pattern was the same as for the upper and lower medians, although the total differences between the curves are greater.

When pocket reduction after treatment was related to oral hygiene scores for pockets in the 4 to $6 \mathrm{~mm}$ range (Fig. 5), there was a slightly greater reduction in pocket depth 1 year after treatment in the group with better than median oral hygiene than in the group with higher plaque scores. This difference was statistically significant for only 4 years postsurgically and the two curves followed a very similar pattern, indicating no significant effect of degree of oral hygiene in maintenance of pocket 
Pocket Depth $1-3 \mathrm{~mm}$

Plaque Score: $\leq 1.32-$ $\geq 2.58--$

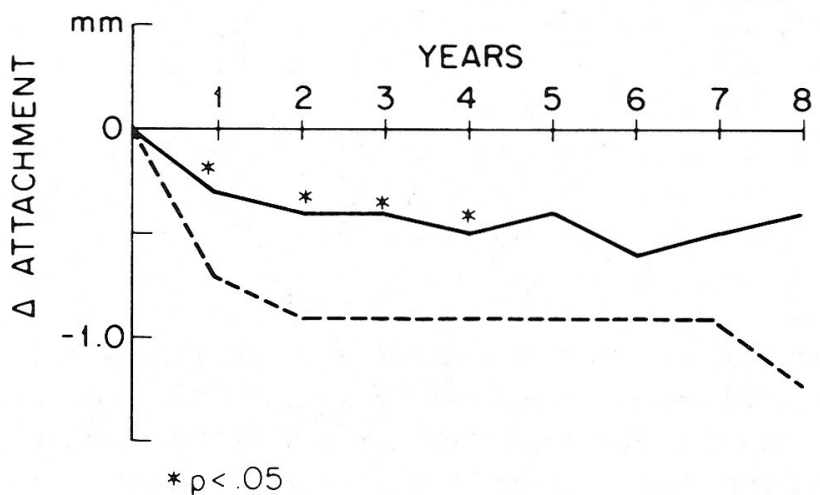

Figure 4. Attachment change related to oral hygiene over 8 years.

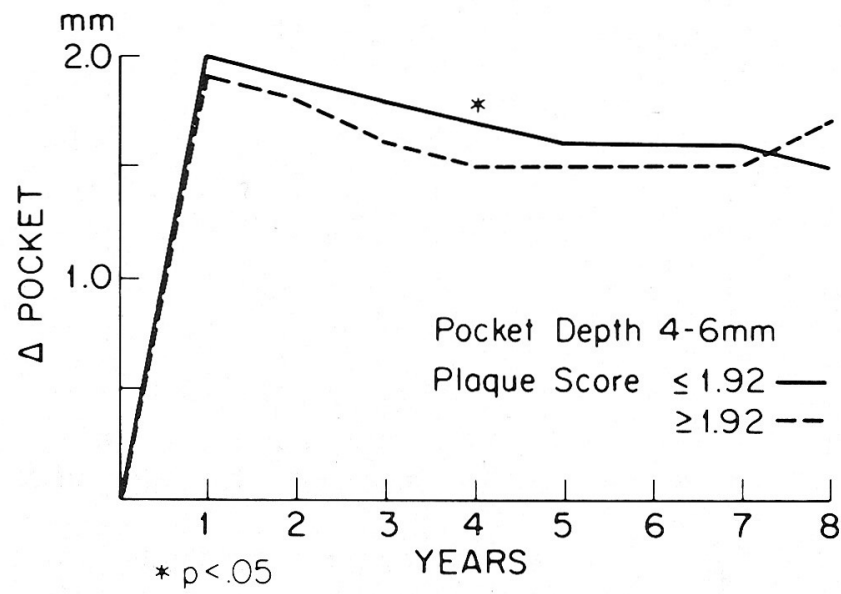

Figure 5. Pocket reduction related to oral hygiene over 8 years.

reduction from 1 year after the initial treatment. Very similar results appeared when reduction in pocket depth for 4 to $6 \mathrm{~mm}$ pockets over time was related to the $25 \%$ lowest and 25\% highest plaque scores (Fig. 6).

Gain and maintenance of clinically measurable attachment for 4 to $6 \mathrm{~mm}$ pockets (Fig. 7) over 8 years were not influenced significantly by the post-treatment plaque scores if the comparison was based on a division of higher or lower than median plaque scores. However, a comparison between the $25 \%$ of individuals having the highest plaque scores and the 25\% having the lowest (Fig. 8), showed a significantly greater gain in attachment for 1 to 4 years in the group with the lowest scores (better oral hygiene). After 4 years the difference was not significant, and the course of the curves from year 1 was very similar.

Pocket reduction related to oral hygiene scores for pockets $\geq 7 \mathrm{~mm}$ over time (Fig. 9) was not consistently related to better or worse than median oral hygiene scores, although there was a tendency in the first 4 years toward less favorable pocket response with higher than median plaque scores. However, this tendency was later reversed, indicating no relationship between return of pocket depth and poorer than median oral hygiene. This lack of relationship became even more obvious when individuals with the highest $25 \%$ of plaque score were compared with the 25\% with the lowest scores (Fig. 10).

While there was a significantly greater gain in clinical attachment in deep pockets for the first 3 years after treatment for the group with better than median oral hygiene (Fig. 11), some of this initial gain was lost over time. Also, maintenance of the attachment levels recorded 1 year after treatment over the subsequent 7 years was fully as good for the high plaque score group as for the low score group. Similar results were apparent when the attachment changes were related to the upper and lowest 25\% plaque score groups (Fig. 12). Although initial post-treatment gain of attachment seemingly is

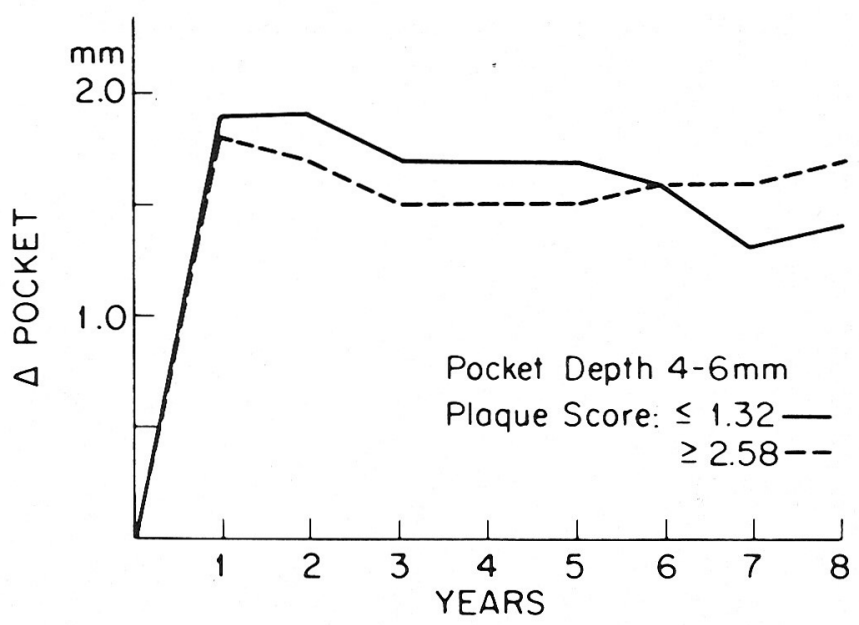

Figure 6. Pocket reduction related to oral hygiene over 8 years.

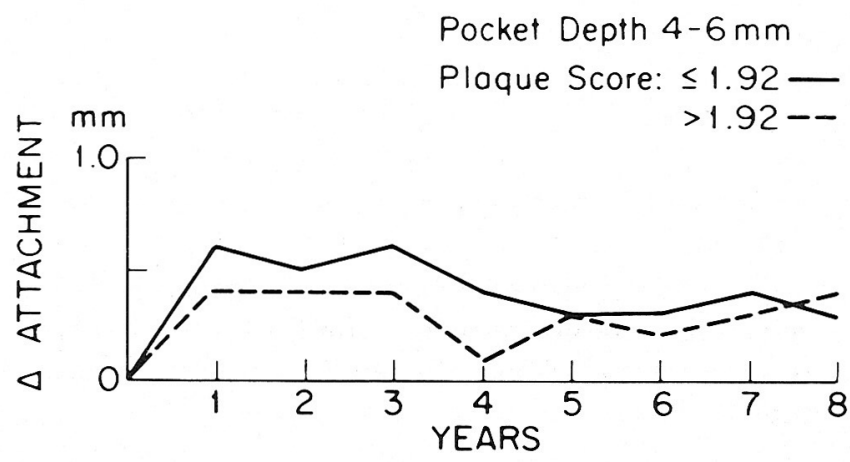

Figure 7. Attachment change related to oral hygiene over 8 years.

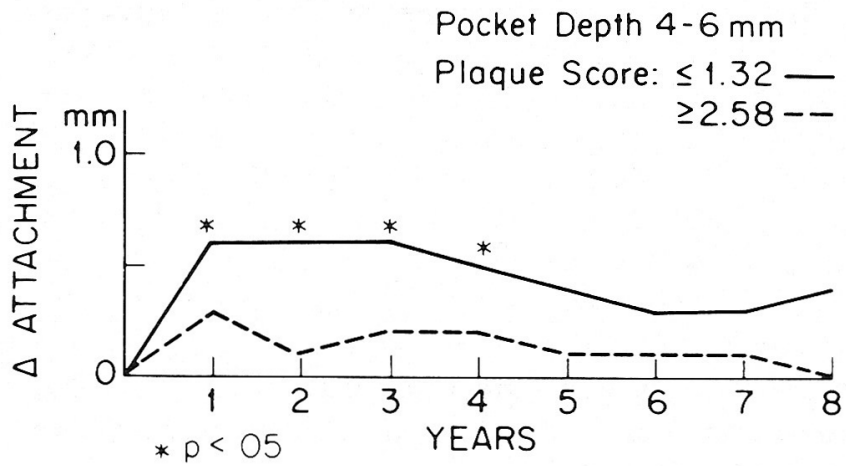

Figure 8. Attachment change related to oral hygiene over 8 years. 


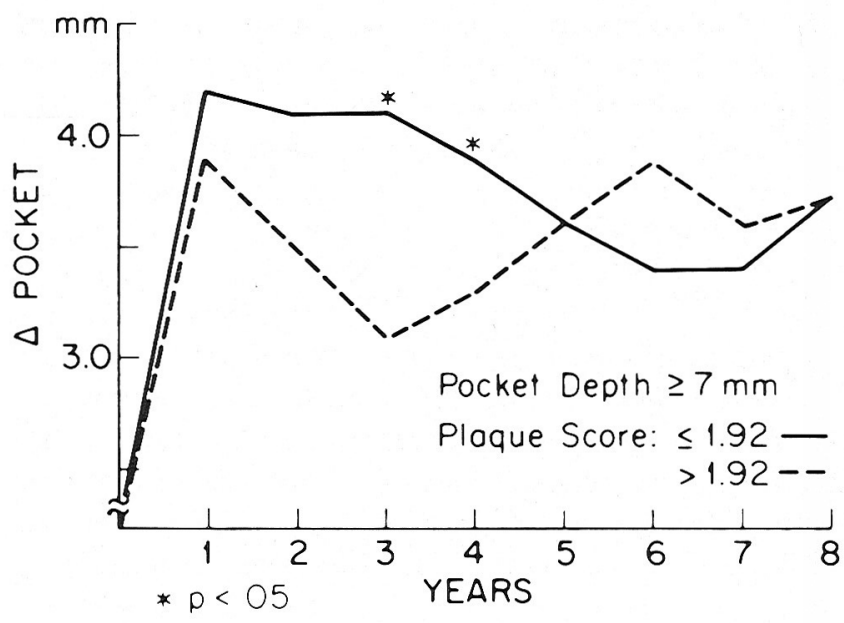

Figure 9. Pocket reduction related to oral hygiene over 8 years.

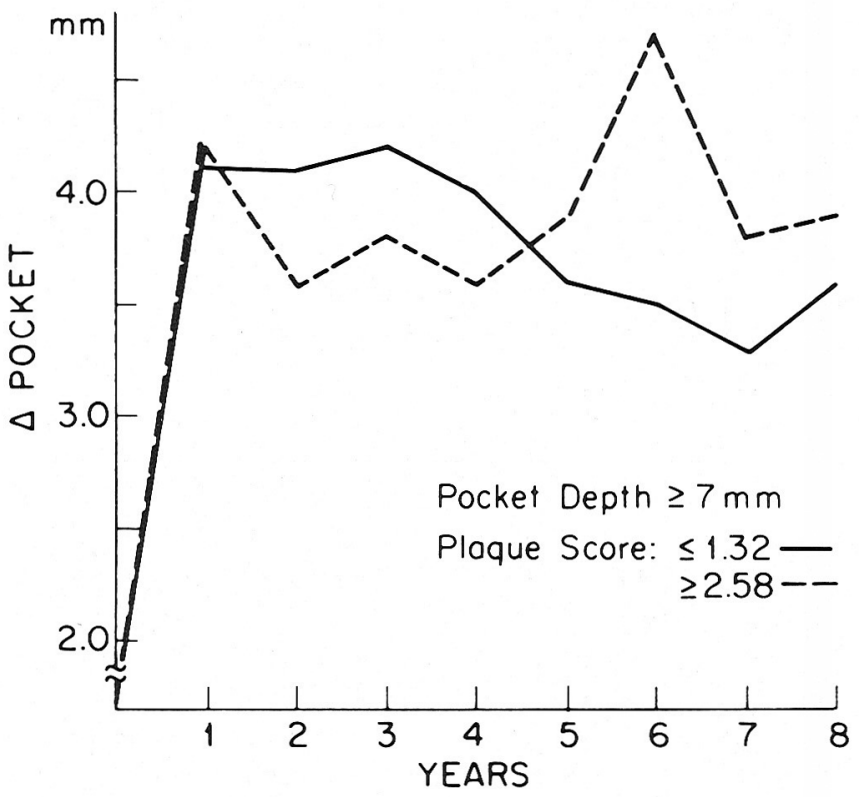

Figure 10. Pocket reduction related to oral hygiene over 8 years.

highly related to the degree of oral hygiene, the longterm results were not dependent on the levels of plaque scores for these patients with recall prophylaxis every 3 months.

\section{SIGNIFICANCE}

It should be emphasized that this is not a study of oral hygiene versus no oral hygiene following periodontal therapy. All of the patients were given repeated instructions and motivational sessions in oral hygiene at each 3-month visit for prophylaxis. All of the patients with initial high plaque scores experienced a considerable reduction associated with the hygienic phase of therapy. ${ }^{20}$ This narrowed the spread of the individual plaque scores. ${ }^{21}$ Although none maintained a zero plaque score there certainly was a great difference from what would have been seen with untreated or completely neglected cases of periodontitis.
The most important finding in this study is that attachment levels and pocket depth 1 year after periodontal treatment can be maintained close to post-treatment levels over 7 more years with prophylaxis every 3 months, regardless of unavoidable variations in the effectiveness of the patient's plaque control.

With longer or shorter recall intervals for professional tooth cleaning, the results might have been entirely different. The results are very comparable to those from similar studies by Axelsson and Lindhe ${ }^{5}$ with 2 to 3 month recall, and should be comforting to the clinician who worries about the patient with less than perfect personal plaque control. The frequent recall seems to prevent destructive effects of newly formed plaque and gingivitis. On the other hand, it was interesting to see that 1 year following periodontal therapy the degree of plaque control tended to influence both the pocket re-

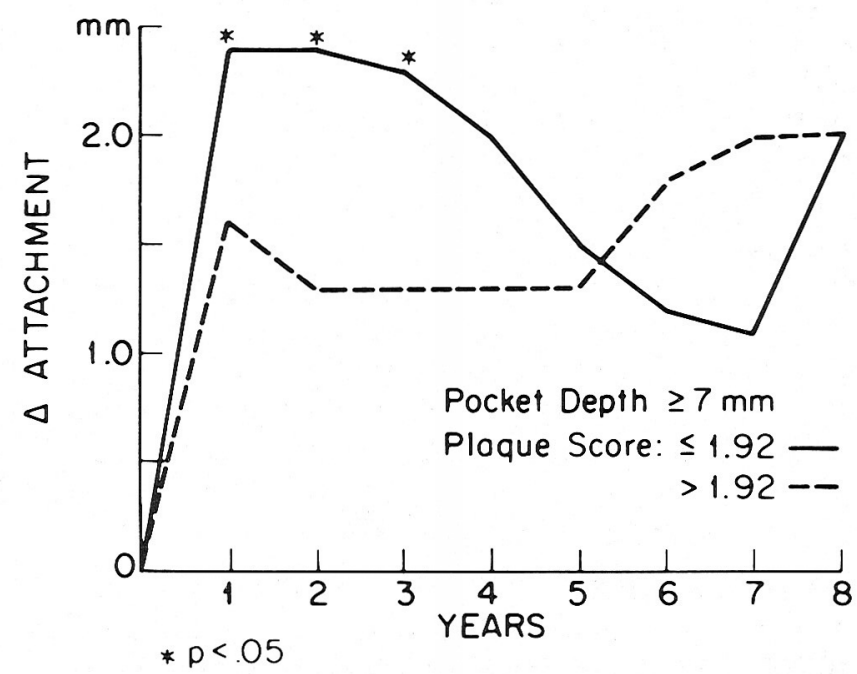

Figure 11. Attachment change related to oral hygiene over 8 years.

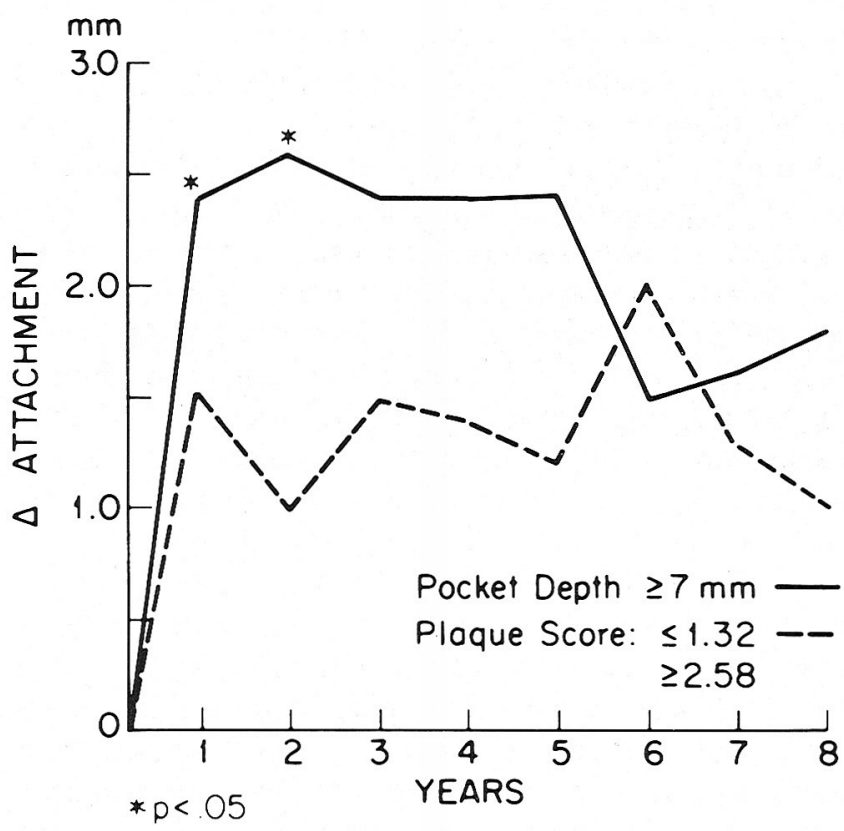

Figure 12. Attachment change related to oral hygiene over 8 years. 
duction and the gain in clinical attachment from the initial baseline. This greater improvement with good oral hygiene seemed to level off over 4 to 5 years, giving equally good long term results for the groups with good or poorer oral hygiene.

From a series of studies recently reviewed by Listgar$\operatorname{ten}^{22}$ it is becoming increasingly evident that the depth of penetration during probing is related to some extent to the inflammatory state of the pocket wall, and it stands to reason that inflammation is reduced less with poor than with good oral hygiene. Thus, the initial results appear to be better in patients with good oral hygiene than in those with poor oral hygiene. However, this does not necessarily mean that the support for the teeth cannot be maintained in spite of less than perfect oral hygiene.

The sudden opening of long epithelial attachments resulting in loss of close tissue adaptation to the teeth has been the subject of speculative concern in the literature. $^{22,23}$ However this does not seem to occur very often since repeated, well controlled measurements over many years of follow-up, ${ }^{5,12}$ with recall every 2 or 3 months, have shown practically unaltered attachment levels and pocket depths. ${ }^{12}$ If anything, there is an average gain in clinical attachment compared with the initial baseline, both shortly after treatment and years later, and this is most apparent for deep pockets. ${ }^{12}$ Thus, probing of attachment levels provides essential information for clinical practice if the concern is to maintain the dentition in a functional state for as long as possible. ${ }^{22}$

\section{CONCLUSIONS}

With professional tooth cleaning every 3 months, the pocket reduction and clinical attachment level gained by therapy can be maintained without significant effect from variations in personal oral hygiene.

\section{REFERENCES}

1. Löe, H., Theilade, E., and Jensen, S. B.: Experimental gingivitis in man. $J$ Periodontol 36: 177, 1965.

2. Lindhe, J., Hamp, S. E., and Löe, H.: Experimental periodontitis in the Beagle dog. J Periodont Res 8: 1, 1973.

3. Ramfjord, S. P.: The periodontal status of boys 11 to 17 years old in Bombay, India. J Periodontol 32: 237, 1961.

4. Löe, H., Aanerud, A., Boysen, H., and Smith, M.: The natural history of periodontal disease in man. The rate of periodontal destruction before 40 years of age. $J$ Periodontol 49: 607, 1978.

5. Axelsson, P., and Lindhe, J.: Effects of controlled oral hygiene procedures on caries and periodontal disease in adults. $J$ Clin Periodontol 5: 133, 1978.
6. Morrison, E. C., Lang, N. P., Löe, H., and Ramfjord, S. P.: Effects of repeated scaling and root planing and/or controlled oral hygiene on the periodontal attachment level and pocket depth in Beagle dogs. I. Clinical findings. J Periodont Res 14: 428, 1979.

7. Lang, N. P., Cumming, B. R., and Löe, H.: Toothbrushing frequency as it related to plaque development and gingival health. $J$ Periodontol 44: 396, 1973.

8. Lindhe, J., and Nyman, S.: The effect of plaque control and surgical pocket elimination on the establishment and maintenance of periodontal health. A longitudinal study of periodontal therapy in cases of advanced disease. J Clin Periodontol 2: 67, 1975.

9. Rosling, B., Nyman, S., and Lindhe, J.: The effect of systematic plaque control on bone regeneration in infrabony pockets. $J$ Clin Periodontol 3: 38, 1976.

10. Nyman, S., Lindhe, J., and Rosling, B.: Periodontal surgery in plaque infected dentition. J Clin Periodontol 4: 240, 1977.

11. Suomi, J. D., et al.: The effect of controlled oral hygiene procedures on the progression of periodontal disease in adults. Results after third and final year. $J$ Periodontol 42: 152, 1971.

12. Knowles, J. W., Burgett, F. G., Shick, R. A., Morrison, E. C., and Ramfjord, S. P.: Results of periodontal treatment related to pocket depth and attachment level. Eight years. J Periodontol 50: 225, 1979.

13. Löe, H., Schiött, C. R., Glavind, L., and Karring, T.: Two years oral use of chlorhexidine in man. I. General design and clinical effects. J Periodont Res 11: 135, 1976.

14. Axelsson, P., and Lindhe, J.: The effect of preventive programe on dental plaque, gingivitis and caries in school children. Results after one and two years. J Clin Periodontol 1: 126, 1974.

15. Socransky, S. S.: Microbiology of periodontal disease. Present status and future considerations. J Periodontol 48: 497, 1977.

16. Listgarten, M. A., Lindhe, J., and Hellden, L.: The effect of tetracycline and/or scaling on human periodontal disease. Clinical microbiological and histological observations. J Clin Periodontol 5: 246, 1978.

17. Syed, S. A., Morrison, E. C., and Lang, N. P.: The predominent dental plaque flora of Beagle dogs following periodontal treatment. $J$ Periodont Res 14: 255, 1979.

18. Ramfjord, S. P.: The periodontal disease index (PDI). J Periodontol 38: 602, 1967.

19. Knowles, J. W.: Oral hygiene related to long-term effects of periodontal therapy. J Mich Dent Assoc 55: 147, 1973.

20. Morrison, E. C., Ramfjord, S. P., and Hill, R. W.: Short-term effects of initial nonsurgical periodontal treatment (hygienic phase). $J$ Clin Periodontol 7: 199, 1980.

21. Zann, G.: Results of periodontal treatment related to gingivitis and oral hygiene over eight years. Thesis, The University of Michigan, Ann Arbor, 1980.

22. Listgarten, M. A.: Periodontal probing: What does it mean? $J$ Clin Periodontol 7: 165, 1980.

23. Armitage, G. C., Svanberg, G. K., and Löe, H.: Microscopic evaluation of clinical measurements of connective tissue attachment levels. J Clin Periodontol 4: 173, 1977.

Send reprint requests to: Dr. S. P. Ramfjord, Department of Periodontics, The University of Michigan, School of Dentistry, Ann Arbor, MI 48109. 